\title{
Highly Intuitive 3-DOF Simultaneous and Proportional Myocontrol of Wrist and Hand
}

\author{
Markus Nowak, Ivan Vujaklija, Claudio Castellini, and Dario Farina
}

\begin{abstract}
While simultaneous and proportional activation of multiple degrees of freedom (DOFs) is supported by novel prosthetic hands, there are still no commercial controllers to appropriately enable it. Here, we test a ridge regression based myocontrol method in two real-time scenarios: 13 subjects with an extended high-density EMG electrode set (192 channels) and 4 subjects with a reduced set of electrodes (16 channels). In each scenario, the algorithm was trained on 3 repetitions of single DOF motions (rest, wrist flexion/extension and rotation, and hand closing) and then subjects were asked to reach 24 on-screen goals consisting of one-DOF, two-DOF, and threeDOF targets. The results showed that participants were able to reach all types of targets and that their one-DOF success rate remained high despite the simultaneous control of multiple DoFs $(95.9 \pm 5.7 \%)$. Moreover, the performance did not significantly change when reducing the number of electrodes $(97.6 \pm 4.5 \%$ for $\mathbf{1 6}$ channels).
\end{abstract}

\section{INTRODUCTION}

Multi-articulate prosthetic hands have been commercially available for over a decade. Even though they feature multiple motorized degrees of freedom (DOFs), the most commonly provided control interface is a switching paradigm leaving users to progressively cycle through preset functions [1]. Recently, advanced prosthetic systems have been controlled with pattern recognition algorithms [2], [3] capable of directly distinguishing individual gestures by classifying electromyographic (EMG) activity of user's residual muscles. While this is an important advance, the lack of simultaneous functions makes it still far from meeting the expectations of natural control.

Enabling simultaneous control over multiple DOFs was attempted by extending the pattern recognition approaches to facilitate simultaneous classes [4]. While feasible, this approach remained bound to laboratory conditions. To overcome the inherent discrete nature of classifiers, a focus has been put on regression approaches which aim to establish a continuous mapping between the observed EMGs and the functional domain of prosthetic joints [5]. However, even after demonstrating that a high number of DOFs can be concurrently controlled in this way in robotic systems using high-density EMG (HD-EMG) [6], the most recent

The study was partially funded by the DFG project Tact_Hand (CA1389/1-1) and the ERC Synergy project NaturalBionicS.

M. Nowak and C. Castellini are with the German Aerospace Center (DLR), Institute of Robotics and Mechatronics, Wessling, Germany (e-mail: \{markus.nowak, claudio.castelini\}@dlr.de).

I. Vujaklija, is with the Department of Electrical Engineering and Automation, Aalto University (e-mail: ivan.vujaklija(aalto.fi).

D. Farina is with the Department of Bioengineering, Imperial College London (e-mail: d.farina@imperial.ac.uk) . home-tested prosthetic systems are still bound to only two DOFs [7].

Here, we propose a regression-based approach which follows the recommendations on being highly intuitive and requiring minimal calibration [8] to deliver 3-DOF simultaneous and proportional control over the wrist and hand (wrist flexion/extension, wrist pronation/supination, and hand closing and rest). The approach was evaluated in real-time and its robustness was examined by progressive channel reduction.

\section{MATERIAL AND MEthods}

\section{A. Control Strategy}

We acquired 192 monopolar EMG signals with three 8-by-8 ELSCH064NM3 sensor matrices (EMG-USB2+ amplifier, OTBioelettronica, Italy), which were placed around the circumference of the forearm. A moving average (MAV) filter was applied to the rectified signal to extract the envelope and a ridge regression (RR) machine learning (ML) method was applied to the EMG envelopes ${ }^{1}$.

\section{B. Experiment Description}

To investigate the combined capability of user and ML method we recruited 13 able-bodied participants (age $27.5 \pm 3$ ). The study was approved by the local ethics committee of Imperial College of London and all participants have given their written consents before participation.

The EMG sensor matrices were placed around the participant's forearm covering the entire circumference. Reference electrodes were placed at the wrist, and subjects were seated comfortably in front of a PC screen (Figure 1).

The subjects performed three repetitions of the aforementioned actions of the wrist and hand at a comfortable level for $2 s$. These $3 \times 6=18$ recordings of the MAV of all available channels were used for training the RR algorithm. Thereafter they performed 24 randomly presented goal reaching tasks (12 one-DOF, 8 two-DOF and 4 three-DOF goals, equally distributed with respect to the origin). These tasks accounted for different levels of activation, e.g. $30 \%$ of wrist flexion combined with $80 \%$ of wrist pronation and $0 \%$ hand closure in a two-DOF goal. The subject had $20 s$ to attempt and reach each goal ( $15 \%$ of the normalised work space), and stay within it for $0.3 s$.

\footnotetext{
${ }^{1}$ The EMG was gathered at $2048 \mathrm{~Hz}$ and treated with a 5 th order Butterworth bandpass-filter $(20 \mathrm{~Hz}-500 \mathrm{~Hz})$. Features were calculated from the rectified, low-pass filtered signal (2nd order Butterworth at $2 \mathrm{~Hz}$ cut-off), and averaged across a window of 100 samples with a $3 / 4$ overlap. The regularization parameter in RR was kept at 1.0.
} 


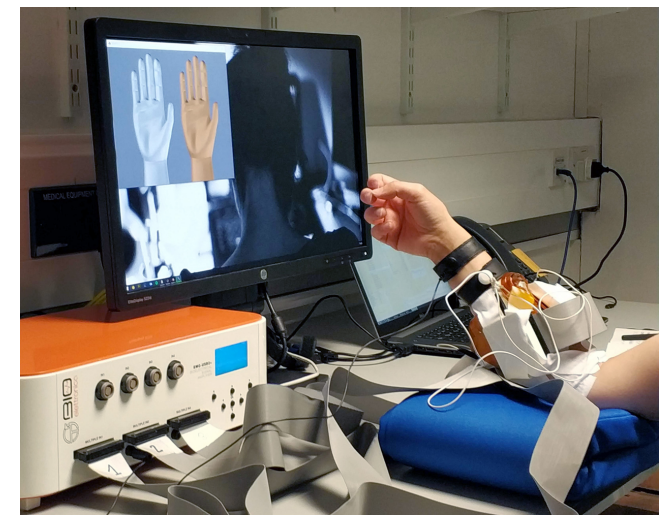

Fig. 1: Experimental setup with $E M G-U S B 2+$ amplifier, PC screen with prediction and target stimulus, and participant equipped with three $8 \times 8$ sensor matrices

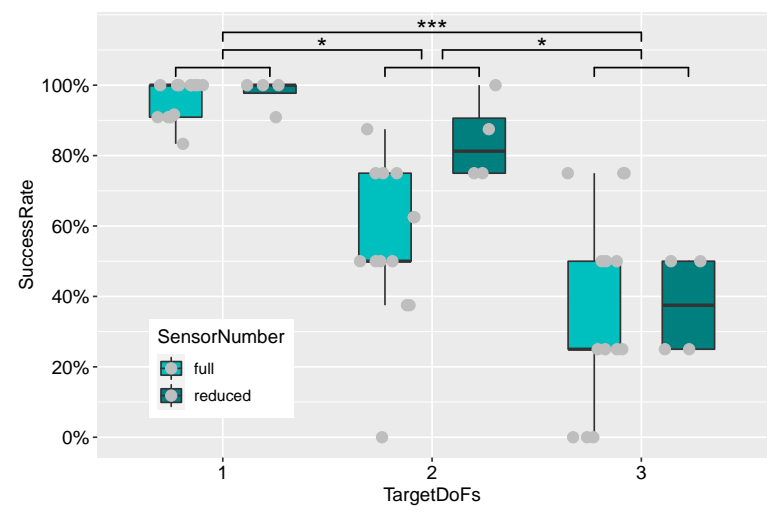

Fig. 2: Box plot with SR of all subjects, superimposed by a dot plot of the individual subject results. Brackets with asterisks represent significant difference, brackets without asterisks represent grouping.

Of the 13 subjects, four completed an additional round of the same 24 tasks, but with a reduced number of EMG sensors - 16 of the original 192 sensors were used for training the ML method. The reduced set featured eight electrode pairs (along the muscle fibre direction) evenly distributed around the widest circumference of the forearm. We did not aim at targeting specific muscle groups.

\section{Statistical Evaluation}

For evaluation purposes we compared the performance of all 192 vs. the reduced 16 sensors. Also, we have considered the difference when dealing with one-DOF, two-DOF and three-DOF goals. Therefore, we calculated the success rate (SR) per subject across these categories and performed a statistical analysis with the two factors Sensor Number [full, reduced] and TargetDOFs [1, 2, 3]. We used a twoway repeated-measures ANOVA with $\alpha=0.05$. Significant interactions were followed up by a pair-wise comparison with Bonferroni correction.

\section{RESUlts}

Figure 2 summarizes the results of the user study in terms of SR per category as well as the results of the statistical analysis. The factor TargetDOF was highly significant with $F(2,6)=30.66, p<10^{-3}$, while the factor Sensor Number showed no significant influence on the outcome measure with
$F(1,3)=0.00, p=1.00$. The interaction term showed no significance either with $F(2,6)=2.51, p=0.16 .^{2}$ A post-hoc of the significant factor TargetDOF revealed that each level is significantly different from another with $p \in[0.018,0.00027]$.

\section{Discussion}

Results show that although the participants have trained the algorithm only with single actions, they were capable of real-time command of two-DOF and even three-DOF combined actions. To the best of our knowledge this is the first time that such a level of control is achieved using intuitive control inputs. Although there was a significant performance degradation when facing more difficult tasks (i.e. two-/three-DOF), this did not impact one-DOF control.

Another remarkable finding is the fact that the channel reduction did not have a significant influence on the performance. A slight, yet not significant, improvement was observed when decreasing the number of EMG channels, which might potentially originate from an increase in signalto-noise ratio for the reduced set.

\section{CONCLUSION}

Here we demonstrated for the first time a successful realtime myocontrol over 3-DOFs in a concurrent and proportional fashion with natural inputs and minimal user and machine training. We achieved this by relying on a simple ML method trained on only individual DOF data, yet the system remained intuitive enough to allow users to achieve simultaneous control by eliciting EMGs of concurrent DOFs. In future, we intend to test this approach with amputee subjects and physical prosthetic systems.

\section{REFERENCES}

[1] I. Vujaklija, D. Farina, and O. C. Aszmann, "New developments in prosthetic arm systems," Orthopedic research and reviews, vol. 8, p. 31, 2016.

[2] COMPLETE CONTROL Handbook, Coapt LLC, Chicago, IL, 2018.

[3] Ottobock, "Technology for people 4.0: Ottobock at OTWorld 2018," https://www.ottobock.com/en/newsroom/news/technology-forpeople-4-0-ottobock-at-otworld-2018.html, 2018, [Online; Accessed: 25-May-2020].

[4] M. Ortiz-Catalan, B. Håkansson, and R. Brånemark, "Real-time and simultaneous control of artificial limbs based on pattern recognition algorithms," IEEE Transactions on Neural Systems and Rehabilitation Engineering, vol. 22, no. 4, pp. 756-764, 2014.

[5] I. Vujaklija, "Novel control strategies for upper limb prosthetics," in International Conference on NeuroRehabilitation. Springer, 2018, pp. 171-174.

[6] M. Ison, I. Vujaklija, B. Whitsell, D. Farina, and P. Artemiadis, "Simultaneous myoelectric control of a robot arm using muscle synergyinspired inputs from high-density electrode grids," in 2015 IEEE International Conference on Robotics and Automation (ICRA). IEEE, 2015, pp. 6469-6474.

[7] J. M. Hahne, M. A. Schweisfurth, M. Koppe, and D. Farina, "Simultaneous control of multiple functions of bionic hand prostheses: Performance and robustness in end users," Science Robotics, vol. 3, no. 19, p. eaat3630, 2018.

[8] C. Castellini, R. M. Bongers, M. Nowak, and C. K. van der Sluis, "Upper-limb prosthetic myocontrol: two recommendations," Frontiers in neuroscience, vol. 9, p. 496, 2016.

\footnotetext{
${ }^{2}$ Sphericity and normality of the residuals were tested with Mauchly's test and with Shapiro-Wilk test, respectively. Furthermore, since repeated measures ANOVA cannot deal with missing data, we additionally performed a multi-level linear analysis, which confirmed the findings.
} 